\title{
THE THERMOELASTIC HERTZ PROBLEM WITH PRESSURE DEPENDENT CONTACT RESISTANCE
}

\author{
Maria Comninou and J. R. Barber \\ Department of Mechanical Engineering and Applied Mechanics, University of Michigan, \\ Ann Arbor, MI 48109, U.S.A.
}

(Received 13 September 1984)

\begin{abstract}
Summary-There is a considerable amount of experimental work establishing that the resistance to heat conduction between two solids in contact depends on the contact pressure, decreasing with increasing pressure. However, there are no solutions of thermoelastic contact problems taking into account this nonlinear behavior. In the present paper, we consider the thermoelastic Hertz contact problem and assume a contact resistance which is inversely proportional to pressure. The solution is compared with those assuming perfect or imperfect contact.
\end{abstract}

\section{INTRODUCTION}

The heat transfer between two solids in contact is of considerable physical and technological interest. A sizable amount of experimental work is devoted to the study of the resistance to heat conduction across the interface between two solids in contact $[1,2,3]$. Since contacting surfaces are not in ideal contact, the presence of asperities leads to contact resistance which decreases with increasing pressure in a nonlinear fashion.

There is little analytical work on the subject, however, and in most thermoelastic contact problems the contact is considered perfect. Under this restriction it has been observed $[4,5]$ that there is no solution satisfying the physical inequalities of the problem for one direction of heat flow, namely when the heat flows into the material with the smaller distortivity. Barber [6] started with an example assuming a pressure dependent resistance and considered the limiting case, which leads to linearised boundary conditons and gives an acceptable solution for the said direction of heat flow. Specifically, his analysis showed that the limiting case corresponds to a zone of 'imperfect' contact located between perfect contact and separation. This new contact zone transmits zero pressure and still provides resistance to heat flow. A series of problems (e.g. [7,8]) has been solved using the idea of imperfect contact.

One significant result that has emerged from this work is that multiple solutions are obtained for both directions of heat flux under certain conditions $[9,10]$. The thermoelastic contact problem with linear or linearised boundary conditions appears beset with questions of existence, uniqueness and even stability of solutions. Some of these questions were tackled by Duvaut [11] in a concise paper which considered the unilateral contact between an elastic solid and a rigid wall assuming pressure-dependent resistance to heat flow. For this problem, and under certain restrictions on the contact resistance, he was able to prove existence and uniqueness.

No solution has so far been obtained, to our knowledge, using the nonlinear boundary condition mentioned. In this paper, we obtain a solution to the plane thermoelastic Hertz contact problem assuming a contact resistance inversely proportional to pressure. Both directions of heat flow are considered.

\section{FORMULATION}

The geometry of the plane Hertz problem is shown in Fig. 1. Two elastic cylinders with different material constants and parabolic profiles are pressed together with a total force $P$. The total heat flux through the contact is denoted by $Q$. The contact interval established under these conditions has unknown extent $2 a$. The formulation is based on a Green's function for interior thermoelastic contact [12]. Details of the preliminary analysis are given in [7], and we record here only the essentials:

Derivative of the gap between the solids

$$
\frac{\mathrm{d} g}{\mathrm{~d} x}=\frac{1}{2 \pi M} \int_{-a}^{a} \frac{p(\zeta) \mathrm{d} \zeta}{\zeta-x}+\left(\delta_{1}-\delta_{2}\right) \int_{-a}^{x} q(\zeta) \mathrm{d} \zeta+K x+B
$$




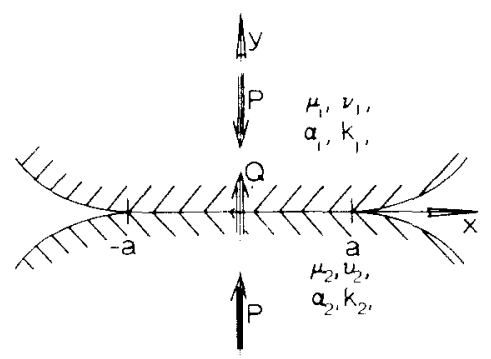

FIG. 1, Geometry of the thermoelastic Hertz contact problem.

Derivative of the temperature jump across the interface

$$
\frac{\mathrm{d} \tau}{\mathrm{d} x}=\frac{1}{\pi} \frac{k_{1}+k_{2}}{k_{1} k_{2}} \int_{-a}^{a} \frac{q(\zeta)}{x-\zeta} \mathrm{d} \zeta .
$$

In these equations $p(x)$ is the contact pressure, $g(x)$ the gap, $\tau(x)$ the temperature jump, $q(x)$ the heat flux at the interface, $K$ is the curvature mismatch and $B$ an arbitrary constant. The material constants $M$ and $\delta$ are given by

$$
\begin{gathered}
M=2 \mu_{1} \mu_{2} /\left[\mu_{1}\left(\kappa_{2}+1\right)+\mu_{2}\left(\kappa_{1}+1\right)\right] \\
\delta=\alpha(1+v) / k,
\end{gathered}
$$

where $\kappa=3-4 v$ for plane strain, $v$ is Poisson's ratio, $\mu$ the shear modulus, $k$ the conductivity and $\alpha$ the coefficient of thermal expansion. Subscripts are used to denote quantities pertaining to the upper or lower solid. Continuity conditions are automatically satisfied with the present formulation, and the only remaining boundary conditions are

$$
\begin{gathered}
\frac{\mathrm{d} g}{\mathrm{~d} x}=0, \quad|x|<a \\
\tau(x)=R(x) q(x), \quad|x|<a \\
R(x)=A / p(x),
\end{gathered}
$$

where $R$ is the contact resistance and $A$ is a constant describing the relation between thermal contact resistance and pressure for the particular material combination and surface condition.

In addition we must satisfy the two auxiliary conditions

$$
\begin{aligned}
& \int_{-a}^{a} p(\zeta) \mathrm{d} \zeta=\mathbf{P} \\
& \int_{-a}^{a} q(\zeta) \mathrm{d} \zeta=\mathrm{Q} .
\end{aligned}
$$

Finally, for a physically meaningful solution, the gap and the contact pressure must be non-negative. The method of solution used in the sequel cannot enforce these inequalities and their validity must be verified a posteriori.

Differentiating (5) and (6), with respect to $x$ and using (1) and (2) we obtain

$$
\begin{gathered}
\frac{\mathrm{d}}{\mathrm{d} x} \int_{-a}^{a} \frac{p(\zeta)}{\zeta-x} \mathrm{~d} \zeta=-2 \pi M\left[K+\left(\delta_{1}-\delta_{2}\right) q(x)\right], \quad|x|<a \\
\frac{1}{\bar{\pi}} \frac{k_{1}+k_{2}}{k_{1} k_{2}} \int_{-a}^{a} \frac{q(\zeta)}{x-\zeta} \mathrm{d} \zeta=A \frac{\mathrm{d}}{\mathrm{d} x}\left[\frac{q(x)}{p(x)}\right],|x|<a .
\end{gathered}
$$

These two singular integral equations must be solved together with (8) and (9). It is possible by manipulating (10) and (11) to obtain a single nonlinear integro-differential equation of the Cauchy type, but there seems to be no advantage in doing so.

\section{NUMERICAL SOLUTION}

To solve the system of equations $[10,11,8,9]$ we adopt a series representation based on Chebyshev polynomials. First we set

$$
x=a \cos \phi, \quad \zeta=a \cos \theta
$$

and then expand $p(\theta)$ and $q(\theta)$ in series

$$
\begin{gathered}
p(\theta)=\sum_{m \text { odd }} p_{m} \sin m \theta, \quad 0<\theta<\pi \\
q(\theta)=\sum_{n \text { odd }} q_{n} \sin n \theta, \quad 0<\theta<\pi .
\end{gathered}
$$


The form of the series guarantees that the heat flux and the pressure will vanish at the ends of the contact interval, and takes into account the symmetry of the problem. Equations (10) and (11) become

$$
\begin{gathered}
\frac{\mathrm{d}}{\mathrm{d} \phi} \sum_{m \text { odd }} p_{m} \int_{0}^{\pi} \frac{\sin \theta \sin m \theta}{\cos \theta-\cos \phi} \mathrm{d} \theta \\
=2 \pi M a \sin \phi\left[K+\left(\delta_{1}-\delta_{2}\right) \sum_{n \text { odd }} q_{n} \sin n \phi\right], \quad 0<\phi<\pi \\
\frac{k_{1}+k_{2}}{k_{1} k_{2}} \frac{1}{\pi} a \sin \phi \sum_{n \text { odd }} q_{n} \int_{0}^{\pi} \frac{\sin \theta \sin n \theta}{\cos \theta-\cos \phi} \mathrm{d} \theta=A \frac{\mathrm{d}}{\mathrm{d} \phi} \frac{\sum_{n \text { odd }} q_{n} \sin n \phi}{\sum_{m \text { odd }} p_{m} \sin m \phi}, \quad 0<\phi<\pi .
\end{gathered}
$$

Observing [13]

$$
\begin{gathered}
\int_{0}^{\pi} \frac{\sin \theta \sin n \theta d \theta}{\cos \theta-\cos \phi}=-\pi \cos n \phi, \quad n=1,2, \ldots \\
\int_{0}^{\pi} \frac{\cos n \theta d \theta}{\cos \theta-\cos \phi}=\frac{\pi \sin n \phi}{\sin n \phi}, n=0,1, \ldots
\end{gathered}
$$

and integrating (17), we obtain

$$
\begin{gathered}
\sum_{\text {modd }} m p_{m} \sin m \phi=2 M a \sin \phi\left[\mathrm{K}+\left(\delta_{1}-\delta_{2}\right) \sum_{n \text { odd }} q_{n} \sin n \phi\right], \quad 0<\phi<\pi \\
\frac{k_{1}+k_{2}}{k_{1} k_{2}} \frac{1}{2} a \sum_{m \text { odd }} \sum_{\substack{n \text { odd } \\
n \neq 1}} q_{n} p_{m}\left[\frac{\cos (n-1) \phi}{n-1}-\frac{\cos (n+1) \phi}{n+1}\right] \sin m \phi \\
=-A \sum_{n \text { odd }} q_{n} \sin n \phi+A C \sum_{m \text { odd }} p_{m} \sin m \phi, \quad 0<\phi<\pi,
\end{gathered}
$$

where $C$ is a constant of integration. Applying the transformation

$$
\int_{0}^{\pi}() \sin r \phi d \phi, \quad r \text { odd }
$$

to (20) and (21) and performing some elementary integrations we obtain

$$
\begin{gathered}
p_{r}=\frac{4 M a}{\pi}\left\{K \frac{\pi}{2 r} \delta_{r 1}+\left(\delta_{1}-\delta_{2}\right) \sum_{n \text { odd }} q_{n}\left[\frac{1}{r^{2}-(n-1)^{2}}-\frac{1}{r^{2}-(n+1)^{2}}\right]\right\}, \quad r \text { odd } \\
\frac{k_{1}+k_{2}}{k_{1} k_{2}} \frac{a \pi}{8}\left[\sum_{\substack{n \text { odd } \\
n \neq 1}} \frac{1}{n-1} q_{n}\left(p_{r-n+1}+p_{r+n-1}\right)-\sum_{n \text { odd }} \frac{1}{n+1} q_{n}\left(p_{r-n-1}+p_{r+n+1}\right)\right]=\frac{\pi}{2}\left(-A q_{r}+A C p_{r}\right), \quad r \text { odd. }
\end{gathered}
$$

We next introduce the following dimensionless constants

and normalize $q_{r}$ and $p_{r}$

$$
\begin{gathered}
\varepsilon_{1}=M a / P \\
\varepsilon_{2}=\frac{A}{M a} \frac{k_{1} k_{2}}{k_{1}+k_{2}} \\
\varepsilon_{3}=Q\left(\delta_{1}-\delta_{2}\right) \\
q_{r}=(Q / a) \hat{q}_{r} \\
p_{r}=(P / a) \hat{p}_{r} .
\end{gathered}
$$

Substituting $(14,15)$ into (8) and (9) yields

$$
\hat{p}_{1}=2 / \pi, \quad \hat{q}_{1}=2 / \pi .
$$

Equations (23) and (24) are now written as

$$
\begin{gathered}
\hat{p}_{r}=\varepsilon_{1}\left(\frac{1}{2} K a \frac{\delta_{r 1}}{r}+\frac{4}{\pi} \varepsilon_{3} S_{2 r}\right), \quad r \text { odd } \\
q_{r}=\frac{1}{\varepsilon_{1}}\left(C-\frac{1}{4} \frac{1}{\varepsilon_{2}} S_{1 r}\right), \quad r \text { odd },
\end{gathered}
$$


where

$$
\begin{gathered}
S_{1 r}=\sum_{\substack{n \text { odd } \\
n \neq 1}} \frac{\hat{q}_{n}}{n-1}\left(\hat{p}_{r-n+1}+\hat{p}_{r+\ldots 1}\right)-\sum_{n \text { odd }} \frac{\hat{q}_{n}}{n+1}\left(\hat{p}_{r-n+1}+\hat{p}_{r+n+1}\right) \\
S_{2 r}=\sum_{n \text { odd }} \hat{q}_{n}\left[\frac{1}{r^{2}-(n-1)^{2}}-\frac{1}{r^{2}-(n+1)^{2}}\right]
\end{gathered}
$$

and the constant $C$ has been redefined.

Terminating the series at $r=N$, equations $(30,31,32.33)$, yield a system of $2(N+1)$ equations for the unknowns $p_{r}, q_{r}, a$ and $C$

The system is solved by iteration as follows: we assume values for $q_{r}(r=3,5, \ldots, N)$, and solve (32) and (30) for $p_{r}$ $(\mathrm{r}=3,5, \ldots, N)$ and $K a$. We then substitute into (33) and solve (33) and (31) for $C$ and the updated values of $q_{r}$. We repeat the process until the solution converges.

\section{RESULTS}

The algebraic sign of the constant $\varepsilon_{3}$ determines the direction of heat flow: a positive sign indicates heat flowing into the material with the larger distortivity, while a negative sign indicates heat flowing in the opposite direction. Representative results are shown in Fig. 2 for both directions of the heat flux. We note that the contact pressure and the heat flux $q(x)$ at the interface vanish at the end-points of the contact. As $\varepsilon_{2}$ decreases, the heat flux develops a peak, as we should expect, since in the limit as $\varepsilon_{2}$ tends to zero we should recover the case of perfect contact in which the heat flux is square root singular at the ends of the contact region. The pressure is affected qualitatively only near the contact ends and for negative $\varepsilon_{3}$.

Figures 3 and 4 show the variation of $K a$ with $\varepsilon_{3}$ and $\varepsilon_{1}$, respectively. We find that $K a$ varies approximately linearly with $\varepsilon_{3}$ and inversely with $\varepsilon_{1}$. A similar relation was obtained in the earlier solution [7]* assuming perfect contact, which in the present notation can be written

$$
K a=\left(\frac{1}{\varepsilon_{1}}-\frac{4 \varepsilon_{3}}{\pi}\right) / \pi .
$$

In fact, we recover the results of [7] in the limit as $\varepsilon_{2}$ tends to zero, and the results show that $K a$ is comparatively insensitive to $\varepsilon_{2}$.

For the opposite direction of heat flow $\left(\varepsilon_{3}<0\right)$, the limit as $\varepsilon_{2}$ tends to zero would be expected to correspond to a solution involving imperfect contact-i.e. a zone of low pressure

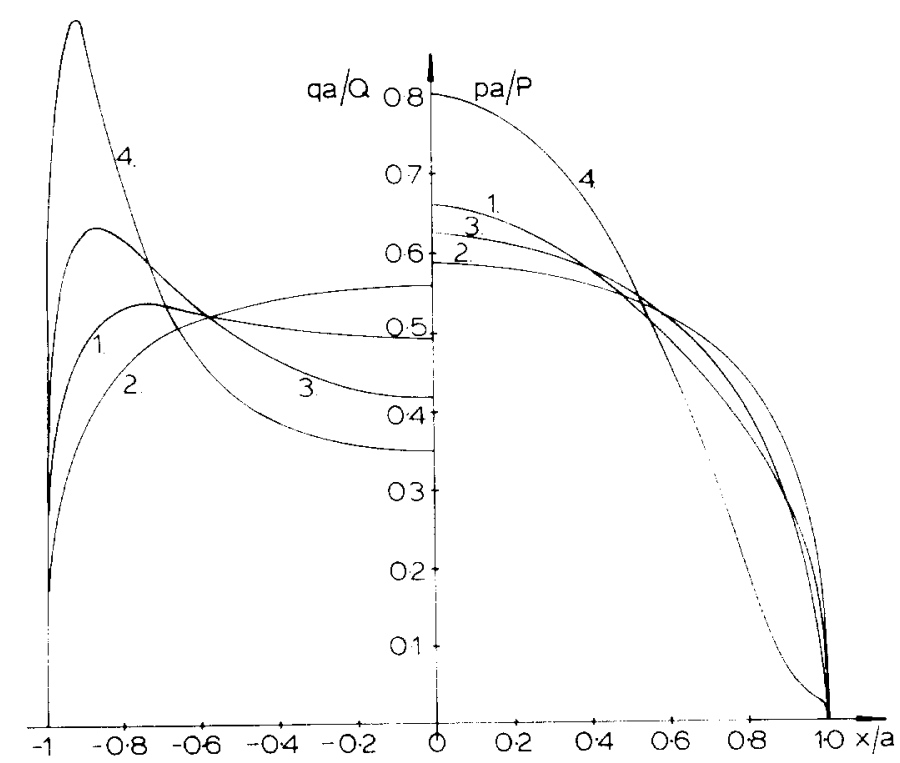

FIG. 2. Representative graphs for heat flux and contact pressure. 1. $\varepsilon_{1}=2 \times 10^{3}, \varepsilon_{2}=0.1 \times 10^{-3}$ $\varepsilon_{3}=-1.5 \times 10^{-3}, 2 . \varepsilon_{1}=1.9 \times 10^{3}, \varepsilon_{2}=1 \times 10^{-3}, \varepsilon_{3}=-1 \times 10^{-3}, 3 . \varepsilon_{1}=1 \times 10^{3}, \varepsilon_{2}=0.1$ $\times 10^{-3}, \varepsilon_{3}=0.18 \times 10^{-3}, 4 . \varepsilon_{1}=2 \times 10^{3}, \varepsilon_{2}=0.01 \times 10^{-3}, \varepsilon_{3}=-1 \times 10^{-3}$

* Equation (31) of [7] contains a misprint. It should read $P / M k a^{2}=\pi+4 Q\left(\delta_{1}+\delta_{2}\right) / \pi K a$. 


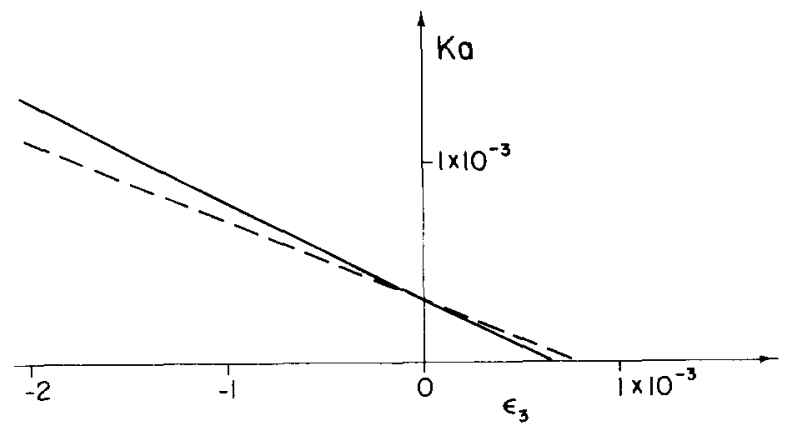

FIG. 3. Variation of $K a$ with $\varepsilon_{3}$ for $\varepsilon_{1}=1 \times 10^{3}, \varepsilon_{2}=0.1 \times 10^{-3}$. Dotted line corresponds to perfect contact $\left(\varepsilon_{2}=0\right)$.

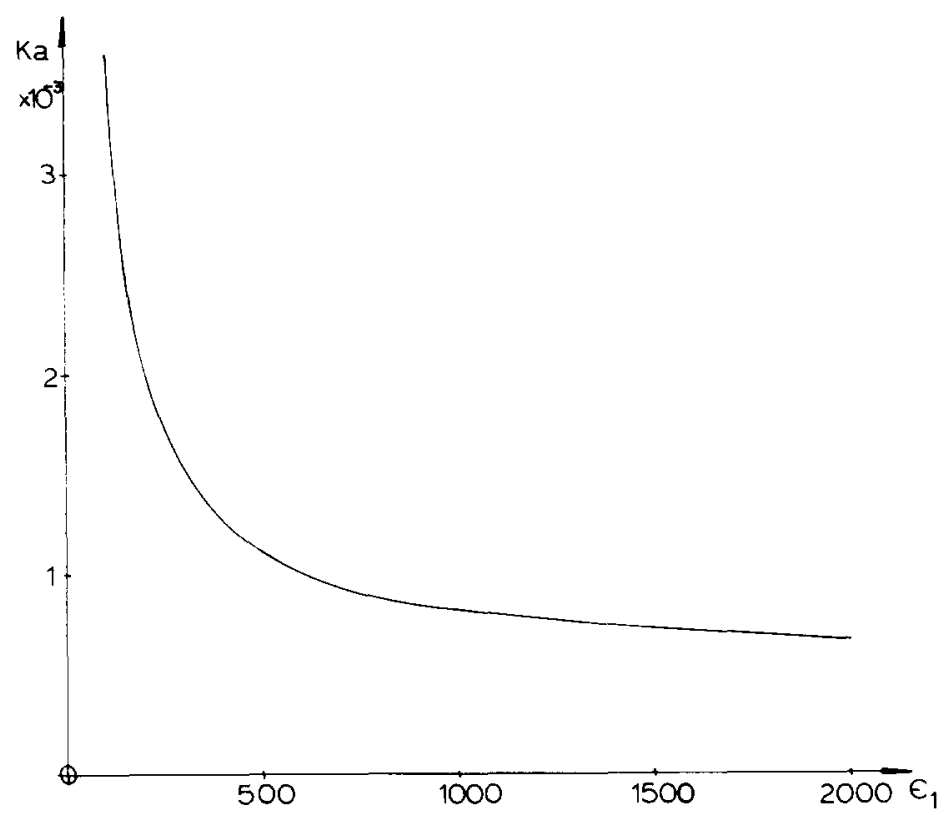

FIG. 4. Variation of $K a$ with $\varepsilon_{1}$ for $\varepsilon_{2}=0.1 \times 10^{-3}, \varepsilon_{3}=-1 \times 10^{-3}$

and high thermal contact resistance-near the ends of the contact region. The pressure profiles in Fig. 2 (curve 4) suggest the development of such a region, but for smaller values of $\varepsilon_{2}$ the solution involves tensile contact tractions. It is probable that another branch of the nonlinear solution does not involve tension, but the region over which this happens is too small to ascertain computationally.

We know from Duvaut's work [11] that there must be at least one physically acceptable solution for all values of $\varepsilon_{2}$, but unfortunately the present iterative formulation does not enable us to force convergence on this solution in preference to that involving tensile tractions.

Acknowledgements-The authors are pleased to acknowledge support by the U.S. Army Research Office under contract DAAG29-82-K-0001.

\section{REFERENCES}

1. M. G. CoOper, B. B. Mikic and M. M. YovanoviCh, Thermal contact conductance, Int. J. Heat MassTrans. 12, 279 (1969).

2. A. M. Clausing and B. T. ChaO, Thermal contact resistance in a vacuum environment, ASME J. Heat Trans. 87, 243-251 (1965).

3. YU. P. ShYLKov and YE. A. GANIN, Thermal resistance of metallic contacts, Int. J. Heat Mass Trans. 7, 243-255 (1964). 
4. J. R. BARber, Indentation of the semi-infinite elastic solid by a hot sphere, Int. J. Mech. Sci. 15, 813-819 (1973)

5. J. Dundurs and C. PANEK, Heat conduction between bodies with wavy surfaces, Int. J. Heat Mass Trans. 19, 73 (1976).

6. J. R. Barber, Contact problems involving a cooled punch, J. Elasticity 8, 409-423 (1978).

7. M. Comninou, J. Dundurs and J. R. Barber, Planar Hertz contact with heat conduction, J. appl. Mech. 48, 549-554 (1981).

8. M. Comninou, J. R. Barber and J. Dundurs, Heat conduction through a flat punch, J. appl. Mech. 871-875 (1981).

9. C. J. Martin-Moran, J. R. Barber and M. Comninou, The penny-shaped interface crack with heat flow-I. Perfect contact, J. appl. Mech. 50, 29-36 (1983).

10. J. R. BARBER and M. Comninou, The penny-shaped interface crack with heat flow-II. Imperfect contact, $J$. appl. Mech. 50, 770-776 (1983).

11. G. Duvaut, Free boundary problem connected with thermoelasticity and unilateral contact, Free Boundary Problems, Vol. II, Pavia (1979).

12. J. Dundurs and M. Comninou Green's function for planar thermoelastic contact problems - interior contact. Mech. Res. Comm. 6, 317-321 (1979).

13. G. M. L. GLADWELL, Contact problems in the classical theory of Elasticity, Sijthoff and Noordhoff, Alphen aan den Rijn, Netherlands (1980). 